\title{
Strates
}

STRATES Matériaux pour la recherche en sciences sociales

9 | 1997

Crises et mutations des territoires

\section{Territoire et exclusion : des mots de l'État- providence et des maux de la société civile}

\section{Catherine Rhein}

\section{(2) OpenEdition \\ 1 Journals}

Édition électronique

URL : http://journals.openedition.org/strates/613

DOI : $10.4000 /$ strates. 613

ISSN : $1777-5442$

Éditeur

Laboratoire Ladyss

Édition imprimée

Date de publication : 30 septembre 1997

ISSN : 0768-8067

Référence électronique

Catherine Rhein, «Territoire et exclusion : des mots de l'État-providence et des maux de la société civile », Strates [En ligne], 9 | 1997, mis en ligne le 19 octobre 2005, consulté le 08 septembre 2020. URL : http://journals.openedition.org/strates/613; DOI : https://doi.org/10.4000/strates.613

Ce document a été généré automatiquement le 8 septembre 2020

Tous droits réservés 


\title{
Territoire et exclusion : des mots de l'État-providence et des maux de la société civile
}

\author{
Catherine Rhein
}

1 Ce texte a une triple ambition, beaucoup plus modeste que son titre : celui de prolonger le débat engagé par Nicole Mathieu en reprenant certains thèmes et en les précisant, celui d'apporter quelques remarques et brèves mises au point en guise de réponse provisoire, enfin celui de passer le relais, en quelque sorte, à d'autres chercheurs. Nicole Mathieu souhaitait que je réagisse sur son texte : j'ai reçu ce texte comme une série d'interrogations de fond, si lourdes qu'il n'est pas question ici d'y répondre de manière vraiment approfondie. Et ce texte se fonde surtout sur les travaux originaux menés par N. Mathieu sur l'exclusion en milieu rural ; donc je reprends ici le relais, en posant d'emblée que l'exclusion est un champ de recherche que je connais mal, mais dont les facteurs qui l'engendrent ne me sont pas inconnus, du moins pour ce qui concerne le champ urbain.

Un peu de sémantique ne nuit point

2 Le terme d'exclusion n'est pas un concept, au sens strict. S. Paugam indique que ce terme est apparu dans les années 1960, dans le cadre de travaux du Commissariat Général au Plan, sous la plume de P. Massé et de l'un des collaborateurs du Père Wresinski1. Puis la publication, en 1974, de l'ouvrage de R. Lenoir, Les Exclus, a joué un rôle-clé dans la diffusion du terme. C'est un terme qui trouve donc son origine dans l'action publique, dans les politiques publiques, plurielles, de lutte contre la pauvreté. En tant que tel, il constitue un premier objet de recherche. En effet pauvretés, marginalités, exclusions sont trois thèmes dont les référents ne coïncident pas exactement, mais qui ont la particularité de désigner tous trois un ensemble de phénomènes sociaux, mais des phénomènes très particuliers, au sens où ils contiennent de la souffrance, de la douleur, du malheur et de l'émotion: ils ne laissent donc pas indifférents, ils émeuvent, ils touchent, ils remettent en question nos positions bien établies de chercheurs ou d'enseignants-chercheurs, inclus parmi les inclus. Aussi 
semble-t-il impossible de parler de la question de l'exclusion sans prendre conscience de deux questions, au préalable.

3 En premier lieu, l'exclusion est un paradigme, construit dans et par l'action de l'Étatprovidence. Ainsi en tant que telle, l'exclusion a une dimension politique incontournable, et cette dimension politique concerne bien la question de la citoyenneté, mais de la façon la plus complète possible : elle concerne tous les niveaux de l'appareil politique, plus encore la société civile dans tous ses replis. Le terme a un effet de catégorisation, qui est nécessairement un effet de stigmatisation. Et ces effets politiques sont pluriels, eux aussi. Ils concernent les structures de classes et remettent notamment en cause l'unité, toute idéologique, d'une classe ouvrière très composite en ce qui concerne ses origines nationales, ses niveaux de revenus, d'éducation et de qualification. Donc user du terme d'exclusion, c'est ipso facto adopter une posture qui est celle de l'État vis-à-vis de certaines parties de la société civile.

4 En second lieu, certains chercheurs font du traitement de l'exclusion une question d'éthique de conviction, voire, pour certains, une ardente obligation. Et une telle position aboutit à des dérapages, parfois très déplaisants. De l'appropriation du thème à la revendication de son exclusivité, l'exclusion est l'un des thèmes de sciences sociales sur lesquels un certain militantisme agressif semble retrouver pied et qui suscite toujours des vocations de Robin des Bois à Superman, voire de charognards qui se font de la misère des autres un bouclier et un instrument pour conquérir de dérisoires lambeaux de notoriété.

5 Enfin, l'exclusion fait l'objet de recherches dans chaque discipline des sciences sociales, mais surtout en sociologie. S. Paugam en a récemment dirigé un bilan, dans lequel il a délibérément contourné les géographes et la géographie, même si la ségrégation spatiale est traitée par le sociologue Y. Grafmeyer comme l'une des dimensions, à la fois incontournable et inessentielle, de l'exclusion.

6 En fait, il y a là, à mon sens, confusion des genres et des termes : division sociale de l'espace, ségrégation résidentielle ou spatiale, exclusion sont des phénomènes qui ne se recouvrent pas, dont les facteurs et les processus ne sont pas nécessairement les mêmes, j’y reviens plus loin. Par ailleurs, S. Paugam m'avait dit, en décembre 1994, n'avoir pas trouvé ce qu'était la contribution de la géographie aux problèmes de l'exclusion; d'ailleurs Y. Guermond se pose la même question sur Cybergéo (Guermond, 1997). Il est clair que si nous éprouvons aujourd'hui le besoin de nous demander, entre géographes, ce que la géographie peut apporter à l'analyse de l'exclusion, il n'y a aucune raison pour que $\mathrm{S}$. Paugam, politologue et sociologue, y réponde à notre place et avant nous, et aille jusqu'à nous proposer des réponses et des solutions que nous ne sommes pas capables de fournir nous-mêmes. On peut aussi adopter une autre perspective : celle de considérer que les travaux portant spécifiquement sur l'exclusion font partie d'un champ un peu particulier, celui des policy studies, du travail social : c'est un champ qui a conquis son autonomie dans un certain nombre d'universités américaines, en restant relativement composite dans ses attaches disciplinaires. En général la sociologie y est dominante, mais l'économie est aussi présente, avec la psycho-sociologie, le droit (social), parfois avec la géographie, mais beaucoup plus rarement.

7 En bref, l'exclusion est une notion qui ne va donc pas du tout de soi. Ainsi, pour avancer, est-il d'abord nécessaire de reculer, c'est-à-dire de déconstruire ces relations entre territoires et exclusion. 
Du territoire et de l'exclusion

8 La question de N. Mathieu sur les rapports entre territoire et exclusion me semble trop globale pour recevoir une réponse satisfaisante. La définition du territoire n'est pas précisée. Ce terme de territoire est, tout comme celui d'exclusion, une construction sociale, historiquement ancrée dans le droit public (Alliès, 1980, pour la France). Où est le bénéfice heuristique de sa transformation en concept ? Je le traque depuis plusieurs années sans l'avoir trouvé. Certains géographes britanniques usent des termes, plus précis et plus efficaces, de locality, area, space, chacun désignant une dimension, une échelle différente de l'espace géographique. Le terme de territoire est polysémique: c'est en cela qu'il est dangereux. Il peut être pris dans un sens très réifié, celui de l'espace - urbain, rural - délimité, circonscrit. Il peut aussi être compris comme espace délimité et géré ou administré, enfin il peut s'entendre comme synonyme de communauté définie comme l'ensemble des personnes, des ménages vivant sur un territoire donné. C'est dans ce sens, le plus riche en apparence, que les confusions sont les plus totales.

Dans la sociologie américaine, notamment dans certains travaux de l'école de Chicago, le terme de communauté a plusieurs sens. Le premier de ces sens est celui de microsociété, le postulat étant que la proximité spatiale est à la fois l'indice de relations sociales et l'inducteur des relations sociales. Le deuxième de ces sens est en fait premier dans la généalogie du terme de communauté : il renvoie à l'un des mythes fondateurs de cette démocratie américaine, celui qui accorde à la démocratie locale, celle qui émane précisément de la vie de la communauté locale, le primat dans la construction, toujours recommencée, de la démocratie américaine. J'y reviens plus loin, en fait aucun des sociologues de l'école de Chicago ne travaille effectivement sur un sens aussi confus du territoire et de la communauté. Dans le cadre des débats sur l'exclusion, les référents de territoire me semblent être principalement les collectivités territoriales servant à la fois d'acteurs dans le traitement de l'exclusion, mais aussi de cadre territorial, les départements et les communes étant les collectivités les plus concernées en la matière.

Et puis il y a ces trop fameux territoires de l'exclusion que seraient les quartiers d'habitat social, sur lesquels types d'habitat, formes de peuplement, statuts d'occupation, politiques publiques et inégalités sociales sont trop souvent confondus.

11 Les quartiers d'habitat social ont un avantage apparent, celui de constituer une unité de gestion pour les gestionnaires et pour les responsables locaux de la politique de la ville. Ce sont aussi, en général, des quartiers facilement repérables sur le plan morphologique. Il est cependant consternant que des chercheurs, ou prétendus tels, se permettent de tout confondre : grands ensembles, habitat social en général, quartiers d'habitat social, immeubles HLM, et de mélanger tous les problèmes - politiques, sociaux, économiques, en les généralisant à l'ensemble du parc, voire à l'ensemble de banlieues dont le statut est totalement imprécis (Vieillard-Baron, 1996). Or les travaux menés dans le cadre de la Délégation Interministérielle à la Ville (D.I.V.), portent un titre pourtant explicite, mais dérangeant : la diversité des quartiers prioritaires : un défi pour la politique de la ville (Champion et Marpsat, 1996). Mentionnée de manière nécessairement euphémisée par J. B. Champion et M. Marpsat, cette grande diversité des situations locales trouve sa source dans le processus éminemment politique de définition des quartiers en convention. La délimitation de ces quartiers est le produit de négociations, le fruit d'un consensus entre acteurs nationaux (D.I.V., administrations centrales des ministères du Travail et de l'Équipement, en particulier) et acteurs locaux 
- Conseils Généraux, municipalités. La partie se joue à plusieurs : le contexte politique est important, mais ce peut être le contexte local qui a l'importance la plus grande.

Prenons un exemple de quartier de convention, celui du quartier des Blagis, situé au sud de Paris et objet de soins tout particuliers à la fois de C. Pasqua, président du Conseil Général des Hauts-de-Seine, et de M. Aubry. Ce quartier est en réalité un assemblage de quatre groupes de logements sociaux, situés chacun dans des portions de quatre communes, mais sur des territoires effectivement contigus : sur Bagneux, dont la municipalité est communiste depuis 1935, sur Fontenay-aux-Roses, dont la municipalité, RPR au moment de la délimitation, est passée au Parti Socialiste en juin 1994, et sur deux communes aux municipalités respectivement RPR (Bourg-la-Reine) et UDF (Sceaux). Sur ces six groupes d'habitat social situés dans le périmètre du quartier de convention, quatre ne relèvent pas, en réalité, du secteur HLM au sens strict, puisqu'il s'agit d'ensembles construits et gérés par la SCIC : les Blagis (Sceaux), La Roue et la Fontaine (Fontenay-aux-Roses) et l'ensemble Tertre-Cuverons, situé sur Bagneux. Il s'agit de groupes de logements très différents les uns des autres, tant par leur morphologie que par leurs histoires, par les modalités de leur peuplement, pour certains anciens déjà, et par les communes et les municipalités dont ils relèvent , plus précisément par leurs formes d'intervention, différentes selon l'orientation partisane. Pourtant ils font bien partie du même quartier de convention.

Pourquoi insister autant sur l'importance du facteur politique dans la délimitation des quartiers? C'est qu'il constitue une dimension fondatrice de ces quartiers, comme secteurs d'intervention publique multidimensionnelle. Gommer cette dimension ou l'euphémiser, cela tient soit au respect du devoir de réserve - tel est le cas de tous les travaux produits dans le cadre de l'INSEE, pour la D.I.V. -, soit à l'adoption, plus ou moins consciente, d'une posture déniant sa dimension politique à l'échelle locale. Pour conclure sur ce point, il me semble qu'il y a là tout un ensemble de recherches à développer, qui n'ont - en apparence - qu'un lointain rapport avec les processus d'exclusion, celui de l'élaboration de la dimension politique dans les politiques publiques dites territorialisées. Et pourtant c'est un champ dans lequel il me semble urgent de nous mettre à la tâche.

Sur l'école de Chicago et sur les rapports entre espace et pauvreté

L'histoire et les travaux de cette école de Chicago restent relativement mal connus en France, notamment en géographie. Un tout premier point est de préciser qu'il existe plusieurs écoles de Chicago : en économie, en architecture et en sociologie. Pour ce qui est de la sociologie, cette école correspond en fait à l'ensemble des jeunes professeurs et des doctorants qui ont produit thèses, ouvrages et articles dans les années 1920 et 1930. En effet, ce département de sociologie de l'Université de Chicago ${ }^{2}$ est fondé en 1892 et sa direction est alors confiée à Albion Small, qui constitue une petite équipe d'enseignants, dont William Thomas, qui mène à bien, avec F. Znaniecki, un ensemble de travaux sur les paysans polonais, en Pologne et aux États-Unis. Incidemment, le passage du milieu rural au milieu urbain, celui de Chicago, constitue l'un des thèmes de ces travaux aussi fréquemment cités que rarement lus, parce qu'inaccessibles ou presque. La publication du Polish peasant s'achève en 1918 et, de manière assez brutale, W. Thomas est sommé de quitter l'université ${ }^{3}$ et une nouvelle équipe, constituée de $\mathrm{R}$. Park et d'E. Burgess, prend le relais.

Ce sont ces deux professeurs qui lancent des dizaines de doctorants sur des sujets de thèse portant sur la société métropolitaine, dans toutes ses dimensions, mais en 
particulier sur les questions d'intégration sociale. Or dans le contexte américain de l'époque, ces questions ne recouvrent pas celles de l'exclusion, sous les formes sous lesquelles nous les concevons aujourd'hui. De fait, il faut distinguer des textes à vocation théorique, pédagogique et programmatique et les travaux de recherche proprement dits. Les textes à vocation théorique et programmatique sont principalement publiés par R. Park et E. Burgess, et dès la fin des années 1920, par Louis Wirth et par R. McKenzie ${ }^{4}$.

Parmi ces textes programmatiques, il en est un qui est souvent cité, mais probablement moins souvent lu, le texte sur la croissance de la ville, publié en 1923 par E. Burgess. C'est dans ce texte que $\mathrm{E}$. Burgess propose ce fameux modèle concentrique, au nom duquel bien des contresens ont été commis ultérieurement. Or ce texte contient des innovations conceptuelles importantes et intéressantes, qui ne sont pas toujours relevées, dans les lectures qui en ont été faites par la suite. Ainsi dans cette analyse de la croissance de la ville, E. Burgess recourt à deux concepts d'espace bien distincts : d'une part, l'espace physique, celui de l'environnement bâti et du parc de logements, d'autre part l'espace social, ou encore l'espace intra-métropolitain au sein duquel se déploie la société urbaine. Chacun de ces espaces a sa propre dynamique, laquelle a ses propres facteurs, dont les rythmes ne coïncident pas avec ceux de l'autre espace. Et le territoire n'est pas une notion utilisée dans ces travaux, pour plusieurs raisons. Dans ces textes programmatiques, mentionnés plus haut, les perspectives se veulent théoriques: les acteurs de la croissance urbaine sont à peine mentionnés, mais l'empreinte des pratiques de zonage est très nette. Plus fondamentalement, ces textes ont pour référent une collectivité territoriale dont les limites sont alors en constant changement, puisque Chicago s'est beaucoup étendu en annexant le territoire d'autres collectivités préexistantes ou des territoires non encore incorporés. Il est important de souligner la différence avec les collectivités territoriales françaises, dont les territoires sont restés inchangés depuis leur définition, à la fin du XVIII siècle : cette capacité des cities à s'étendre en fonction de leurs besoins n'a aucun équivalent en France, puisque les agglomérations urbaines, telles que les définit l'INSEE, ne sont que des agrégats statistiques de communes aux limites inchangées et intangibles : ce point sur la gestion territoriale est assez éloigné de la question de l'exclusion, mais il est central dans celle du territoire, évidemment.

Pour revenir à l'exclusion, il ne me paraît pas juste de poser que l'école de Chicago s'est interrogée sur ses relations avec le territoire et s'est penchée sur l'exclusion. D'abord, je viens de le montrer, la notion de territoire est très différente, aux États-Unis, de ce qu'elle est en France. Ensuite, dans les autres travaux, c'est-à-dire dans les recherches, dans les thèses et dans les nombreux travaux sous contrat menés pour la ville de Chicago ou pour ses différentes institutions, la question majeure est celle des processus d'intégration sociale, pour les jeunes, pour les immigrants de fraîche date ou plus anciens, pour les ruraux et pour les urbains. Il y a bien certes des processus d'exclusion dans ce Chicago des années 1920 et 1930, mais il s'agit soit d'une exclusion temporaire, frappant les immigrants d'origine européenne, soit d'une exclusion aux racines toutes différentes, frappant les Africains-Américains. Or de cette exclusion-là, il n'est alors pas possible de parler frontalement, directement, explicitement, en pleine période Jim Crow, celle au cours de laquelle la ségrégation et la discrimination sévissent le plus durement $^{5}$, même si le système Jim Crow concerne exclusivement les états du Sud. Donc les sociologues du département de sociologie de l'Université de Chicago travaillent beaucoup sur la délinquance et sur les gangs - il y a alors de quoi faire, d'autant qu'il 
s'agit souvent de commandes, là encore -, mais aussi sur la figure du $\mathrm{Hobo}^{6}$, sur le ghetto juif de Chicago, plus fantasmé que réel ${ }^{7}$, sur la taxi-dance girl, mais aussi sur les Beaux Quartiers, c'est-à-dire sur la Gold Coast ${ }^{8}$, enfin sur les structures et les dynamiques du secteur immobilier'. Bref, à scruter les titres des recherches alors menées, il se dégage plutôt une impression de foisonnement, de très grande richesse thématique ; et ces travaux permettent simultanément des avancées méthodologiques fondamentales, sur les différentes techniques d'enquêtes en particulier, mais aussi en cartographie et en statistique appliquée. Certes il existe un point commun à toutes les recherches : c'est la ville de Chicago, comme terrain et comme objet, mais c'est aussi et surtout la société américaine, telle qu'elle s'inscrit dans Chicago, qu'elle y évolue, s'y forge, s'y façonne et s'y recompose. La dimension politique n'est qu'en apparence absente de ces travaux : elle y resurgit constamment, mais implicitement. Il faut en effet lire ces recherches en gardant constamment présent à l'esprit le jeu de contraintes sous lequel écrivaient leurs auteurs : le taudis s'efface alors, pour céder la place au ghetto noir, en pleine structuration, comme l'a démontré T. Philpott (1978), mais il ne pouvait être question de l'évoquer, pour les sociologues de Chicago. Il ne sera possible de le faire que pour la troisième génération de chercheurs, notamment pour 0 . D. Duncan et B. Duncan, dans les années 1960. Mais ceci est une autre histoire.

Pour conclure sur cette école (de sociologie) de Chicago, elle compte actuellement plus de cinq générations de travaux ; l'écologie humaine n'en constitue qu'une petite partie, au statut étrang ${ }^{10}$. L'écologie factorielle urbaine n'a pas de lien très direct, ni avec cette écologie humaine, ni avec cette école de sociologie de Chicago, de façon plus générale. Il s'agit d'un courant méthodologique, sinon théorique, qui connut son heure de gloire dans les années 1970 ; ce sont les géographes, bien plus que les sociologues qui ont pratiqué cet usage très particulier de l'analyse factorielle. A l'heure actuelle, l'écologie factorielle urbaine est peu pratiquée, et il en va aussi bien ainsi. Il est simplement dommage que l'on n'ait pas mieux souligné combien la structure même des variables $^{11}$ induit plus de problèmes qu'elle ne permet d'en résoudre, tant les interactions sont importantes entre variables, mais difficiles à déceler et à maîtriser. L'accès à des données beaucoup plus fines - par exemple les fichiers-sources des recensements de population - permet de résoudre une partie de ce problème. C'est donc aujourd'hui que l'analyse factorielle, surtout l'analyse des correspondances, simple ou multiple, pourrait être utilisée avec beaucoup de profit, comme technique exploratoire et pré-modélisatrice ; il est dommage qu'avec l'eau de l'écologie factorielle urbaine, on ait jeté celle des techniques multidimensionnelles, mais ceci est encore une autre histoire.

Enfin, dernier point : cette écologie factorielle urbaine a été pratiquée en géographie française, au début des années 1970, par deux équipes notamment, la première étant le groupe Dupont, l'autre étant une équipe strasbourgeoise comprenant E. Dalmasso, H. Nonn et M. Pruvot. Dans le laboratoire STRATES, cette démarche n'a pas été utilisée, du moins dans l'équipe Espace-population-société : nous avons en revanche abondamment utilisé l'analyse factorielle des correspondances, sur des tableaux de données complexes, en particulier dans l'important travail mené sur les traductions spatiales des processus ségrégatifs en Île-de-France (Rhein, 1987 ; Berger et Rhein, 1988 ; Rhein, 1991), mais d'une façon très (sans doute trop) discrète. Il s'agit là d'un autre débat, méthodologique celui-là: sur ce point, la géographie urbaine manque d'acquis 
équivalents à ceux existant en sociologie : c'est donc aussi un autre chantier sur lequel le travail doit s'intensifier.

Ségrégation résidentielle et exclusion dans les grandes métropoles : quelques pistes de recherche

Il m'apparaît que la géographie urbaine a beaucoup à dire sur l'exclusion, sans pour autant que le thème soit central (et il n'a pas à l'être) et elle parle déjà beaucoup de ces processus dans les travaux nord-américains, néerlandais et britanniques ${ }^{12}$. C'est dans les processus de production de l'exclusion que la contribution de la géographie urbaine est à la fois riche en acquis et pleine de potentialités, notamment en ce qui concerne les marchés du travail, les marchés du logement et les structures des couches sociales.

Quelques résultats de travaux sur la division sociale de l'espace me semblent constituer à la fois des acquis et des points de départ pour de nouvelles recherches. Ainsi l'analyse de l'évolution des structures d'emploi indique la diminution des emplois les moins qualifiés - ouvriers et employés - à différentes échelles, de celle de l'ensemble de l'îlede-France à celle de la ville de Paris. La désindustrialisation impliquait, depuis les années 1970, un solde négatif d'emplois ouvriers; depuis les années 1980, ce sont les postes d'employés qui sont affectés, en particulier ceux d'employés administratifs, les plus féminisés. Il faudrait faire le tour des métropoles françaises pour évaluer l'ampleur des dégâts actuels et futurs. En effet, entre 1982 et 1990, ces postes ont augmenté, à l'échelle de la France entière, passant de 6,2 millions à 6,9 millions; leur diminution en valeurs absolue et relative dans la métropole parisienne doit-elle être interprétée comme le résultat d'un processus de décentralisation géographique de tels emplois, ou comme le signe de tendances plus profondes, qui affecteront, à terme, l'ensemble des structures d'emploi, à l'échelle nationale? La conséquence de telles évolutions est claire dans la production de l'exclusion : les moins qualifiés, ceux qui ne parviendront pas à des niveaux de qualification de niveau 2 ou 3 au minimum ne trouveront plus de travail, de quoi vivront-ils? De quoi pourront vivre les très nombreuses mères de famille qui, du jour au lendemain, doivent entrer sur le marché du travail à la suite d'un veuvage ou d'un divorce, sans qualifications et sans expérience? Jusqu'alors, ce sont des postes d'employées qu'elles occupaient, mais qu'en sera-t-il dans dix ans? (Rhein, 1996).

En ce qui concerne le marché du logement, l'une des raisons majeures de la croissance des sans-abri est la disparition, engagée depuis plus de vingt ans, d'un parc social de fait, constitué de garnis, d'hôtels meublés, de logements de fonction comme les loges de concierge d'immeubles industriels ou d'habitation. Dans la ville de Paris, ce stock de logements était très important, puisqu'il représentait encore près d'un logement sur cinq au début des années 1970. Depuis lors, ce parc a fondu de plus de moitié. Il est d'ailleurs important d'analyser le degré d'ouverture ou de clôture des différents marchés du logement, à Paris, dans ses banlieues et sur ses marges. Ainsi, le marché banlieusard est un marché beaucoup moins ouvert aux jeunes entrant sur le marché du logement et aux personnes en situation précaire, si l'on mesure ce degré d'ouverture par la proportion de logements en location libre et de ce stock social de fait. Le parc social (de droit) est, par contraste, un parc particulièrement fermé, au sens où les procédures de sélection sont complexes, les conditions d'admission rigides et parfois discrétionnaires, enfin les délais d'accès en général assez longs. Les espaces de liberté sont, à Paris, de plus en plus ténus : si la flexibilité du travail augmente fortement, au contraire, le marché du travail s'est nettement raidi. 

d'analyse dans lequel plusieurs résultats importants ont déjà été dégagés. En premier lieu, la croissance des ménages constitués de personnes actives vivant seules est l'évolution la plus marquée, en particulier parmi la catégorie des cadres et celle des employés. On se préoccupera peu de l'évolution des catégories les plus aisées, sauf à indiquer que l'un des changements sociaux majeurs a consisté en une gigantesque substitution de cadres à des ouvriers et des employés dans les arrondissements centraux de Paris, ceci à structures de ménage équivalentes, c'est-à-dire de personnes vivant seules. En effet, le parc de logements de Paris-ville fonctionne, pour moitié, comme parc d'accueil de jeunes actifs entrant à la fois sur le marché du travail et sur le marché du logement, qu'ils soient d'origine provinciale ou banlieusarde. Cette fonction de pompe aspirante et refoulante est très ancienne: elle devient de plus en plus sélective socialement.

Une autre évolution, très préoccupante, est celle du taux d'activité des conjointes. $\mathrm{Ce}$ taux est désormais aussi élevé (71\%) parmi les couples biactifs dont les chefs sont des cadres que parmi ceux dont les chefs sont des ouvriers, et c'est dans cette dernière catégorie qu'il a le moins progressé au cours de la dernière période intercensitaire. C'est une évolution préoccupante, puisque la biactivité des conjoints constitue l'une des meilleures protections contre les risques de chômage. Or ce risque est maximal pour la catégorie des ouvriers. A cela, deux raisons : la première est que les ouvriers sont de plus en plus étrangers, la seconde tient à cette diminution des postes d'employées, occupés par la majorité des conjointes d'ouvriers. Le taux d'activité des conjointes d'ouvriers étrangers est significativement plus faible que celui des conjointes d'ouvriers français, pour des raisons culturelles et socio-économiques analysées ailleurs par M. Guillon; le problème est qu'actuellement, ces ménages ayant pour chef des ouvriers étrangers sont donc à la fois les familles les plus nombreuses, celles vivant le plus souvent d'un seul salaire, et les plus exposées au risque de chômage.

De façon plus générale, l'analyse des couches sociales doit être menée de manière globale, au sens où il est important de travailler sur l'intégralité de ces structures sociales: l'accroissement des inégalités ne peut être évalué autrement. Par ailleurs cette analyse des couches sociales permet d'aborder plusieurs dimensions - sociales, politiques, économiques - des processus de ségrégation et d'exclusion.

Ces quelques éléments, très concrets et très résumés, avaient seulement pour prétention d'indiquer que l'analyse des facteurs des processus d'exclusion sont, en milieu urbain, relativement bien identifiés. Il faut bien sûr approfondir l'analyse, l'étendre à d'autres métropoles, dépasser la rupture entre milieux urbains et ruraux. Sur ce dernier point, j'ai proposé de reprendre l'analyse des couches sociales et des structures de ménages dans des espaces de référence plus larges et il faudrait que ce projet prenne forme et aboutisse. Donc j'espère avoir montré qu'il ne me semble pas qu'il y ait des oppositions de fond, juste des violons théoriques et méthodologiques à accorder.

27 En conclusion, je propose donc d'ouvrir ce débat, en dépassant cette distinction entre milieux urbains et ruraux - car il ne me semble pas qu'il s'agisse d'une véritable opposition - et en ouvrant aussi les frontières, c'est-à-dire en se proposant de développer des approches comparatives. C'est un champ de recherches sur lequel, à l'échelle européenne, il existe déjà une littérature abondante et de très grande qualité : nous connaissons mal ces travaux, par exemple ceux de G. Esping-Andersen sur les 
différents types d'États-providence et sur les diverses formes d'exclusion qu'ils engendrent (Esping-Andersen, 1990 et 1993). Dans un champ un peu différent, le débat sur l'underclass, qui s'est développé autour des travaux de W. Julius Wilson aux ÉtatsUnis, a constitué une façon d'euphémiser la question africaine-américaine (ou, plus brutalement, raciale): il rebondit actuellement en Europe en des termes à la fois différents et pourtant proches aussi (Musterd, 1994). Là encore, il faut d'urgence se décider à participer à ce débat, ne pas attendre qu'il vienne à nous, à la fois par les directives européennes, par les appels d'offres et par les travaux des géographes néerlandais, britanniques et belges, en particulier.

\section{BIBLIOGRAPHIE}

Alliès P. (1980), L'invention du territoire, Grenoble, Presses Universitaires de Grenoble, 186 p.

Berger M. \& Rhein C. (1988), « Logements et ménages : une grille pour révéler la division sociale de l'espace », STRATES, 3, pp. 85-130.

Brun J. \& Rhein C. dir.(1994), La ségrégation dans la ville, concepts et mesures, L'Harmattan, 250 p.

BULMER M. (1984), The Chicago school of sociology : institutionalization, diversity and the rise of sociological research, Chicago, University of Chicago Press, 285 p.

Champion J.B. \& Marpsat M. (1996), « La diversité des quartiers prioritaires : un défi pour la politique de la ville », Economie et Statistique, n²94-295, pp. 47-65.

COULON A. (1992), L'École de Chicago, Paris, Presses Universitaires de France, Que Sais-je ? n²639.

Dogan M. \& S. Rokkan S. eds. (1969), Quantitative ecological analysis in the social sciences, Cambridge, Ma., M.I.T. Press, 2 vol.

Esping-Andersen G. (1990), The three worlds of Welfare capitalism, Cambridge, Polity Press.

Esping-Andersen G. (1993), Changing classes : stratification and mobility in post-industrial societies, London, Sage.

Grafmeyer Y. \& Joseph I. (1979), L'École de Chicago, Paris, Eds Champ Urbain-Aubier, rééd. 1990.

Guermond Y. (1997), « Have geographers anything to say about exclusion? », Cybergéo, Hot Cakes.

Kennedy S. (1996), Introduction à l'Amérique raciste, Ed. de l'Aube (rééd. de l'ouvrage publié en 1954 en français, avec une préface de J.P.Sartre, puis en 1989 aux États-Unis sous le titre Jim Crow guide).

Lepetit B. \& Topalov C. dir. (1998), La Ville dans les sciences sociales, à paraître aux éditions de l'EHESS.

Musterd S. ed., (1994), « A rising European underclass? », Built Environment, vol.20, n³, special issue.

PAUGAM S., dir. (1996), L'Exclusion, l'état des savoirs, Paris, La Découverte, 583 p. 
Philpott T.L. (1978), The slum and the ghetto, neighborhood deterioration and middle-class reform, Chicago, 1880-1930, New-York, Oxford University Press, 428 p.

RHEIN C.(1987), Transformations des couches sociales et dynamique du marché de l'emploi dans la première couronne de banlieue de Paris, 1954-75, Paris, rapport de recherche, ATP du CNRS Travail, emploi et conditions de travail, 1987, $120 \mathrm{p}$.

RHEIN C. (1991), « Formes, facteurs et processus de la division sociale de l'espace dans l'agglomération centrale de Paris », pp. 164-272 in STRATES, Traductions spatiales des processus ségrégatifs en Ile-de-France, Paris, CNRS-Direction Régionale de l'Equipement Ile-de-France.

RHEIN C. (1996), « Social segmentation and spatial polarization in Greater Paris », p. 45-70 in J. O'Loughlin \& J. Friedrichs, eds., Social polarization in post-industrial cities, Berlin-New-York, Gruyter/Aldine, 1996.

Vieillard-Baron H. (1996), Les Banlieues, Paris, Flammarion-Dominos.

\section{NOTES}

1. p.9 in Paugam, 1996.

2. Il existe plusieurs universités à Chicago, en particulier Northwestern University, fameuse aussi. University of Chicago est la première université fondée à Chicago, il s'agit d'une université privée.

3. Ce départ tient à des raisons qui ne présentent aucun intérêt ici. Pour les lecteurs qui souhaitent en savoir un peu plus sur l'école de Chicago, voir Coulon (1992), comme introduction ; pour ceux qui veulent en savoir beaucoup plus, il faut consulter en priorité Bulmer (1984) ; la bibliographie de Coulon est bien faite et très nourrie, je me permets d'y renvoyer.

4. Je signale que ces deux derniers auteurs ont beaucoup publié sur la question du continuum urbain-rural : une bibliographie critique est en cours d'élaboration et devrait être présentée dans le cadre du séminaire sociologie/géographie de l'U.M.R. LADYSS au cours de l'année 1997-1998. Certains de ces textes fondateurs ont été édités en français par Y. Grafmeyer et I. Joseph (1979).

5. Sur le système Jim Crow, qui concerne surtout les états du Sud, voir Kennedy (1996).

6. 11 s'agit de la thèse de N. Anderson; c'est un ouvrage passionnant, que l'on peut enfin lire en français, grâce à la traduction d'O. Schwartz.

7. Voir L. Wirth, Le Ghetto, traduit en 1980 par P.J. Rojtman et publié aux éditions du Champ Urbain.

8. Il s'agit des Beaux Quartiers de Chicago, situés au nord du Central Business District, au bord du lac Michigan.

9. II s'agit de la thèse de Ph.D. d'Everett Hughes, dont les travaux ultérieurs seront très différents : sur ce point, J.M. Chapoulie propose, dans le $n^{\circ} 27$ de Sociétés contemporaines, un dossier complet sur Hughes.

10. Je me permets de renvoyer, sur ce point, à mon chapitre sur la ségrégation et ses mesures, in Brun et Rhein (1994) et à celui que j'achève actuellement, sur le Ghetto de L.Wirth, qui sera publié in B. Lepetit et C. Topalov (1998).

11. En écologie factorielle urbaine, les variables portent sur des populations (au sens statistique) très différentes : individus, ménages, population active, logements, collectivités territoriales, sans hiérarchie, contrairement à ce qui se faisait à la même période en analyse écologique (Dogan et Rokkan, 1969). 
12. Une bibliographie sur les grandes métropoles est en cours de préparation, dans le cadre de l'atelier sur la division sociale de l'espace (GDR Socio-Economie de l'Habitat, $n^{\circ} 1100$, PIR-Villes, CNRS, et Plan Construction-Architecture), par C. Rhein, E. Sabot et S.C. Guitton.

\section{RÉSUMÉS}

Dans ce texte qui répond aux réflexions de Nicole Mathieu publiées dans ce même numéro, sont développés trois points. En premier lieu, il est proposé un bref retour aux origines et au sens à donner à la question de l'exclusion. Puis sont abordés les rapports entre territoire et exclusion en milieu urbain, notamment dans les travaux de l'école de sociologie de Chicago. Enfin plusieurs pistes de recherches sont proposées sur les processus et les facteurs d'intégration/exclusion sociales dans les grandes métropoles.

\section{Territory and exclusion}

This paper is an answer to N. Mathieu's paper published in the same issue. In the first place, the very notion of exclusion and its origins are examined. Then the relations between urban territory and exclusion are put under question, in particular in the works of the Chicago school of sociology. Finally a research agenda is set up, regarding factors and processes of social exclusion in post-industrial metropoles.

\section{INDEX}

Mots-clés : exclusion sociale, intégration sociale, ségrégation urbaine, politiques sociales, métropoles, espace urbain, société urbaine

Keywords : social exclusion, social polarisation, social integration, urban segregation, residential segregation, welfare policies, post-industrial metropoles, urban society

\section{AUTEUR}

\section{CATHERINE RHEIN}

Catherine RHEIN, chargée de recherches au CNRS (LADYSS), travaille sur la division sociale de l'espace dans le grand Paris et sur les dimensions politiques et socioéconomiques de ce processus. Dans le cadre du GDR Socio-Economie de l'Habitat, elle est responsable d'un atelier sur les relations entre organisation spatiale de la production et division sociale de l'espace. 